\title{
Enhanced Neural Responses to Imagined Primary Rewards Predict Reduced Monetary Temporal Discounting
}

\author{
(DShabnam Hakimi ${ }^{1 \star}$ and TTodd A. Hare ${ }^{2,3 *}$ \\ ${ }^{1}$ Computation and Neural Systems and ${ }^{2}$ Humanities and Social Sciences Divisions, California Institute of Technology, Pasadena, California 91125, and \\ ${ }^{3}$ Laboratory for Social and Neural Systems Research, Department of Economics, University of Zurich, 8006 Zurich, Switzerland
}

\begin{abstract}
The pervasive tendency to discount the value of future rewards varies considerably across individuals and has important implications for health and well-being. Here, we used fMRI with human participants to examine whether an individual's neural representation of an imagined primary reward predicts the degree to which the value of delayed monetary payments is discounted. Because future rewards can never be experienced at the time of choice, imagining or simulating the benefits of a future reward may play a critical role in decisions between alternatives with either immediate or delayed benefits. We found that enhanced ventromedial prefrontal cortex response during imagined primary reward receipt was correlated with reduced discounting in a separate monetary intertemporal choice task. Furthermore, activity in enhanced ventromedial prefrontal cortex during reward imagination predicted temporal discounting behavior both between- and within-individual decision makers with $62 \%$ and $73 \%$ mean balanced accuracy, respectively. These results suggest that the quality of reward imagination may impact the degree to which future outcomes are discounted.
\end{abstract}

Key words: decision making; fMRI; neuroeconomics; temporal discounting

\section{Significance Statement}

We report a novel test of the hypothesis that an important factor influencing the discount rate for future rewards is the quality with which they are imagined or estimated in the present. Previous work has shown that temporal discounting is linked to individual characteristics ranging from general intelligence to the propensity for addiction. We demonstrate that individual differences in a neurobiological measure of primary reward imagination are significantly correlated with discounting rates for future monetary payments. Moreover, our neurobiological measure of imagination can be used to accurately predict choice behavior both between and within individuals. These results suggest that improving reward imagination may be a useful therapeutic target for individuals whose high discount rates promote detrimental behaviors.

\section{Introduction}

Individuals frequently face choices between options with immediate benefits and alternatives that only yield rewards later in the future, and these decisions can have important consequences for overall well-being. When faced with such trade-offs, humans and other organisms show a tendency to discount the value of delayed rewards (Ainslie, 2001; Green and Myerson, 2004), a phenomenon referred to as temporal or delay discounting. Studies of tem-

\footnotetext{
Received May 14, 2015; revised Aug. 13, 2015; accepted Aug. 20, 2015.

Author contributions: S.H. and T.A.H. designed research; S.H. and T.A.H. performed research; S.H. and T.A.H. analyzed data; S.H. and T.A.H. wrote the paper.

We thank Antonio Rangel for helpful comments and discussions on this work

The authors declare no competing financial interests.

*S.H. and T.A.H. contributed equally to this work.

Correspondence should be addressed to Dr. A. Todd Hare, Laboratory for Social and Neural Systems Research,

Department of Economics, University of Zurich, Blümlisalpstrasse 10, 8006 Zurich, Switzerland. E-mail: todd.hare@econ.uzh.ch.

DOI:10.1523/JNEUROSCI.1863-15.2015

Copyright $\odot 2015$ the authors $\quad 0270-6474 / 15 / 3513103-07 \$ 15.00 / 0$
}

poral discounting have shown that there is considerable variation across individuals in the tendency to postpone gratification to obtain larger, delayed rewards (Kirby and Maraković, 1995; Green and Myerson, 2004; Radu et al., 2011; van den Bos and McClure, 2013). Moreover, differences in the rate at which future rewards are discounted have been linked to measures of general intelligence (Shamosh et al., 2008), financial stability and physical health (Moffitt et al., 2011), as well as the propensity for addiction and substance abuse (MacKillop et al., 2011).

One hypothesis suggests that individual variation in discount rate may be due in part to differences in representing the value of future rewards. The vivid imagination of future episodes could contribute to intertemporal choice (ITC) by allowing individuals to experience an event's (undiscounted) reward value at the time of decision, thereby facilitating self-control behavior. Recent behavioral and neuroimaging studies have addressed the role of imagery and episodic prospection in ITC tasks (Berns et al., 2007; Suddendorf and Corballis, 2007; Benoit et al., 2011; Peters and Büchel, 2011; Daniel et al., 2013; Lebreton et al., 2013). For ex- 
ample, Peters and Büchel (2010) demonstrated in a delay discounting task that participants discounted future rewards less when presented offers paired with self-generated episodic events, and that the magnitude of the effect increased with participants' imagery scores. At the neural level, this effect was represented in the ventromedial prefrontal cortex (vmPFC) as well as other brain regions. In another study, choices made after imagining spending a future payoff were associated with greater vmPFC activity and showed significantly attenuated temporal discounting (Benoit et al., 2011). Together, these data demonstrate that enhancing reward imagery at the time of choice activates prefrontal valuation networks and reduces observed discounting rates. Moreover, in addition to the role of vmPFC in temporal discounting and, more generally, goal-directed choice (Bartra et al., 2013; Clithero and Rangel, 2014), activity in this region has also been reported to increase when explicitly imagining rewards (Bray et al., 2010).

In the present study, we conducted a novel test of the hypothesis that the imagination or simulation of rewarding experiences is associated with temporal discounting rates by comparing the difference in vmPFC activity levels for imagined versus experienced juice rewards to discount rates in a separate monetary ITC task. Here, the comparison between imagined and experienced rewards treats the level of activity during consumption as a baseline value from which positive or negative deviations are measured during reward imagination; we therefore used the relative level of BOLD activity in the vmPFC during reward imagination as an objective measure of imagined reward representation rather than relying on participants' self-reported imagination ability. Specifically, we examined whether relatively greater activity while imagining rewards would be associated with reduced temporal discounting. The studies mentioned above have all shown that manipulations of imagery at the time of choice may influence discount rates. We tested a more general link between reward imagination and temporal discounting by examining whether a neural measure of primary reward consumption imagery could be used to make out-of-sample predictions about delay discounting behavior for a separate class of rewards at a different point in time.

\section{Materials and Methods}

Participants. Thirty healthy individuals were included in the analyses presented here (22 males; mean age, 24.2 years; age range, $19-40$ years). (Six other participants were excluded because their experimental data were corrupted or their choice patterns did not allow for accurate estimation of the hyperbolic discounting parameter, $k$; one additional participant was excluded from the experiment because of excessive head movement [ $\geq 2 \mathrm{~mm}$ in translation or rotation]). All participants had normal or corrected-to-normal vision, no history of neurological, psychiatric, or metabolic illness, and were not taking any medications that interfere with the BOLD signal at the time of scanning. Participants earned $\$ 45$ for participation in the study (which included the juice reward imagination and monetary ITC tasks), an additional $\$ 5$ for arriving on time, and up to an additional \$54 depending on choices made in the ITC task. The Institutional Review Board at California Institute of Technology approved the methods and procedures used in this study.

Monetary ITC task. On each trial, participants were instructed to choose between earning a smaller amount of money today and a larger amount at some time in the future. The smaller, sooner offer was always $\$ 25$, while the larger, later offer ranged from $\$ 25$ to $\$ 54$; the delays ranged from 7 to $200 \mathrm{~d}$ in the future (Hare et al., 2014). The exact combination of offers and delays can be seen in Table 1. Analyses of the fMRI data collected during the monetary ITC task were previously reported by Hare et al. (2014); those fMRI data were not, however, used to test the separate hypotheses addressed in this manuscript. All participants made choices
Table 1. Amounts by delay for the ITC task ${ }^{a}$

\begin{tabular}{rllllll}
\hline Delay (days) & \multicolumn{3}{l}{ Amounts (US \$) } & & & \\
\hline 7 & 25 & 26 & 28 & 30 & 32 & 35 \\
10 & 25 & 26 & 27 & 29 & 30 & 32 \\
12 & 25 & 26 & 28 & 31 & 33 & 35 \\
14 & 25 & 26 & 28 & 32 & 35 & 39 \\
21 & 26 & 27 & 29 & 30 & 32 & 38 \\
25 & 27 & 29 & 31 & 33 & 35 & 46 \\
28 & 26 & 28 & 32 & 35 & 39 & 46 \\
30 & 26 & 27 & 29 & 30 & 32 & 38 \\
40 & 27 & 33 & 35 & 40 & 47 & 54 \\
45 & 26 & 29 & 31 & 35 & 40 & 46 \\
50 & 27 & 30 & 35 & 40 & 46 & 54 \\
60 & 29 & 33 & 35 & 40 & 47 & 54 \\
90 & 26 & 30 & 33 & 40 & 46 & 54 \\
95 & 31 & 33 & 35 & 40 & 47 & 54 \\
100 & 26 & 31 & 38 & 39 & 46 & 54 \\
150 & 31 & 33 & 35 & 40 & 47 & 54 \\
180 & 27 & 31 & 35 & 39 & 46 & 54 \\
200 & 26 & 28 & 35 & 39 & 47 & 54 \\
\hline
\end{tabular}

${ }^{a}$ This table represents the matrix of exact combinations of monetary amounts (US \$) and delays (days) presented to each subject.

over the same set of offer combinations. This feature of the design is important because it allowed us to compute a general, model-free measure of temporal discounting that simply reflected the frequency of choosing the delayed over the immediate reward.

In addition to this model-free measure, we estimated individual discount factors (denoted by $k$ ) for each participant using maximum likelihood procedures under the assumption that participants assigned value to the delayed options using a hyperbolic discounting function. Specifically, the value of a dollar amount, $A$, at a delay of $D$ days is given by the following:

$$
d S V=A /(1+k D)
$$

where $d S V$ denotes the present discounted value of the stimulus (i.e., monetary amount). We also assumed that the probability of accepting the delayed option is given by the soft-max function as follows:

$$
P(\text { Yes })=(1+\exp (b *(25-d S V)))^{-1}
$$

where $b$ is a non-negative parameter that modulates the slope of the psychometric choice function. In this formula, the value of the constant reference option is $\$ 25$.

Juice reward imagination task. Upon arriving for the experiment, participants reported their relative preference for three fruit juices (apple, grape, or fruit punch) and their current level of thirst. Participants were instructed to refrain from consuming any liquids beginning $3 \mathrm{~h}$ before the experiment, and each participant's most preferred juice was used during the subsequent neuroimaging experiment. The experiment involved the imagined (Imagine) or real consumption (Consume) of both water and the preferred juice inside the MR scanner. Water and juice were randomly assigned to either a blue triangle or an orange square; these shapes served as cues that identified the type of liquid that would be delivered in each trial. After receiving detailed instructions, participants were allowed several practice trials to become familiar with receiving liquid rewards in the scanner.

At the onset of each trial, a stimulus that consisted of the colored shape cue surrounded by either a light gray rectangle (Consume) or thought bubble (Imagine) appeared onscreen for $4.22 \mathrm{~s}$. To ensure that they were attending to the task, participants were instructed to press a button as soon as they recognized the cue. In Consume trials, the delivery of $0.75 \mathrm{ml}$ of liquid was initiated at trial onset and reached the mouth after a delay of $\sim 200 \mathrm{~ms}$. Participants were instructed to "fully taste the liquid" for the duration of the cue. In Imagine trials, no liquid was delivered. Nonetheless, participants were instructed to behave as they did in the Consume trials and to imagine tasting the liquid for the duration of the cue. In both conditions, participants were instructed to swallow either the real or 
imaginary liquid when the word "swallow" appeared onscreen. Each trial was followed by a fixation period (mean $=6 \mathrm{~s}$; range $=4-8 \mathrm{~s}$ ). There were 120 trials in total (30 per action condition) divided into eight experimental blocks. Each block of either Imagine or Consume was comprised of 15 mixed trials with both juice and water cues. Both block order and stimulus presentation order within blocks were randomized across participants.

Following the fMRI session, participants completed a questionnaire to assess their perceived level of success in the task. Using a 10-point, anchored scale $(1=$ "not successful at all," $5=$ "somewhat successful," $10=$ "extremely successful"), each individual reported how successful he or she felt at imagining tasting the liquids overall and separately for water and juice. Additionally, participants were asked to report how similar the experiences of imagining and consuming were both overall and separately for each liquid, where $1=$ "not similar at all," $5=$ "somewhat similar," and $10=$ "extremely similar." Similarity ratings were not obtained for one participant.

Imaging data acquisition. Functional imaging data were collected using a Siemens 3.0 tesla Trio MRI scanner. Using an eight-channel, phased array head coil, we collected gradient echo T2*-weighted EPI images with BOLD contrast. To optimize BOLD sensitivity, we used a tilted acquisition in an orientation $30^{\circ}$ oblique to the anterior-posterior commissure line (Deichmann et al., 2003). The imaging parameters were as follows: $\mathrm{TR}=2500 \mathrm{~ms} ; \mathrm{TE}=30 \mathrm{~ms}$; flip angle $=80^{\circ} ; \mathrm{FOV}=192 \mathrm{~mm}$; in-plane resolution $=3 \mathrm{~mm} \times 3 \mathrm{~mm}$; and 403 - $\mathrm{mm}$ slices $(0.3 \mathrm{~mm}$ gap) with ascending, interleaved acquisition. The first four volumes of each run were discarded to allow for sufficient time for the magnetic field to achieve steady-state magnetization. Task (i.e., monetary ITC or juice reward imagination) order was counterbalanced across participants, and each participant completed the juice reward imagination task over two scanning runs (285 volumes per run). High-resolution, whole-brain T1weighted structural images $(\mathrm{TR}=1500 \mathrm{~ms}$; $\mathrm{TE}=3.05 \mathrm{~ms}$; flip angle $=$ $10^{\circ}$; voxel resolution $=1 \mathrm{~mm}^{3}$; single-shot, ascending acquisition) were also collected for each of the participants. These images were coregistered with their respective EPI images to assist with the anatomical localization of the functional activations.

fMRI data preprocessing. Imaging data were preprocessed using SPM8 (Wellcome Department of Imaging Neuroscience, Institute of Neurology, London) toolboxes run in MATLAB (version 2012a, The MathWorks). Data were corrected for motion with realignment to the mean image, spatially normalized to the MNI EPI template, resampled to 3 $\mathrm{mm}^{3}$ voxels, and spatially smoothed using a Gaussian kernel (FWHM $=$ $8 \mathrm{~mm}$ ). Data were also temporally filtered to remove low-frequency drifts using a high-pass filter cutoff of $128 \mathrm{~s}$.

fMRI modeling and data extraction. We estimated a GLM with AR(1) for each individual. We specified five regressors, one for each of the possible trial types (i.e., R1, Consume Water; R2, Consume Juice; R3, Imagine Water; R4, Imagine Juice) and one for all Swallow (R5) trials. All events were modeled with their full stimulus duration (4.22 s for R1-R4 and $1 \mathrm{~s}$ for R5) and convolved with SPM8's canonical hemodynamic response function. Motion parameters and session constants were also included as regressors of no interest.

To test the hypothesis that the representation of imagined relative to consumed rewards in vmPFC is associated with individual differences in temporal discounting, we extracted parameter estimates for the contrast, (Imagine Juice - Consume Juice), from an ROI defined by a separate and independent study on imagined and real rewards (Bray et al., 2010). The vmPFC ROI was defined as a sphere (radius $=10 \mathrm{~mm}$ ) centered on the coordinates (MNI $x, y, z=0,33,-21)$. The temporal discounting parameters were not normally distributed; therefore, all correlation analyses were conducted using permutation-based tests that generated the null distribution from 10,000 permutations of the data. CIs for all correlation coefficients were estimated from 10,000 bootstrap samples of the data. Our a priori hypothesis was that, relative to actual reward consumption, enhanced imagination of rewards would lead to lower temporal discounting rates. For this reason, we used one-sided tests for negative correlations with measures of imagination (behavioral and neural) and discounting rates. All other correlations without a priori predictions about the sign of the relationship were assessed using two-sided tests. We conducted power analyses to determine the required sample sizes for different effect sizes ( $r$ coefficient) at a significance level of $\alpha=0.05$ (one sided) and a power level of 0.80 . These analyses showed that sample sizes of 23 or 37 would be required to detect significant correlations with an $r=0.5$ and $r=0.4$, respectively. The final sample size of 30 represented a reasonable compromise between statistical power needed to examine individual differences and the experimental costs associated with MRI.

After finding a significant correlation between vmPFC activity for imagined juice rewards and monetary temporal discounting rates, we further tested the generalizability of this relationship using an out-ofsample classification analysis. Because the data were selected for both tests on the basis of previous and independent results (Bray et al., 2010), there is no issue of circularity between our correlation and classification analyses. The classification algorithm we used was a simple GLM as follows:

$$
\mathrm{Y}=\beta_{0}+\beta_{1} * \text { Con_vmPFC }+\varepsilon
$$

where $\mathrm{Y}$ is the temporal discount rate $(k)$ for each participant in the training set, $\beta_{0}$ is the intercept, and $\beta_{1}$ parameterizes the relationship between temporal discount rates and the contrast for imagining versus consuming juice in vmPFC (Con_vmPFC). In each iteration of crossvalidation, the GLM was estimated on 28 participants (training set), and then the $\beta_{1}$ parameters were used to predict discount rates for each of the two participants in the test set. These predictions were then used to classify one test participant as the higher and the other as the lower temporal discounter. This procedure was repeated for each of 435 unique divisions of our 30 participants in training sets of 28 and test sets of two. We then computed the posterior distribution of the classifier's balanced accuracy using a Bayesian approach with a uniform prior on accuracy over the interval $(0,1)$ (for full details, see Brodersen et al., 2010). Briefly, the balanced accuracy approach uses the confusion matrix, in which the rows represent the true labels and the columns represent the predicted labels generated by the classification results. The balanced accuracy is computed as follows:

$$
\frac{1}{2}\left(\frac{\mathrm{TP}}{\mathrm{P}}+\frac{\mathrm{TN}}{\mathrm{N}}\right),
$$

where the variables $\mathrm{TP}$ and $\mathrm{TN}$ represent the number of correctly predicted positives and negatives, respectively, $\mathrm{P}$ represents the actual number of positives, and $\mathrm{N}$ represents the actual number of negatives in the sample. Computing the balanced accuracy in this fashion controls for any imbalance in the data classes that may bias classifier accuracy.

Last, we tested for any bias in our classification approach by computing the accuracy of a set of 1000 classifiers in which the correspondence between the discount rates and vmPFC activity was disrupted by randomly reshuffling the discount rates across participants in the training sets.

We further assessed the predictive power of the juice imagination measure by testing how well the imagination-derived, out-of-sample estimates of discount rate predicted choices on every trial for each participant. In the classification scheme above, each participant was paired with every other participant in the sample, resulting in a total of $29 k$ values estimated from imagination-related vmPFC activity based on different training sets of 28 participants; we computed a participant-specific $k$ from the median over these 29 estimates. Next, we computed the present discounted value of each delayed reward by substituting the imaginationderived $k$ into the hyperbolic discounting equation described previously. Using these parameters, choices for the delayed reward were predicted whenever the present discounted value was greater than the $\$ 25$ available on the day of the experiment. Prediction accuracies were evaluated using the same balanced accuracy measure cited in the previous paragraph. Last, we compared the choice prediction accuracy obtained using $k$ parameters estimated from neural activity during juice imagination to the number of choices correctly predicted by a benchmark model based on the group's choice behavior. (The benchmark model used a $k$ computed separately for each of the 30 participants as the median of the $k$ parameters estimated from the choice data of the other 29 participants.) This benchmark model represents an out-of-sample prediction mechanism 
a

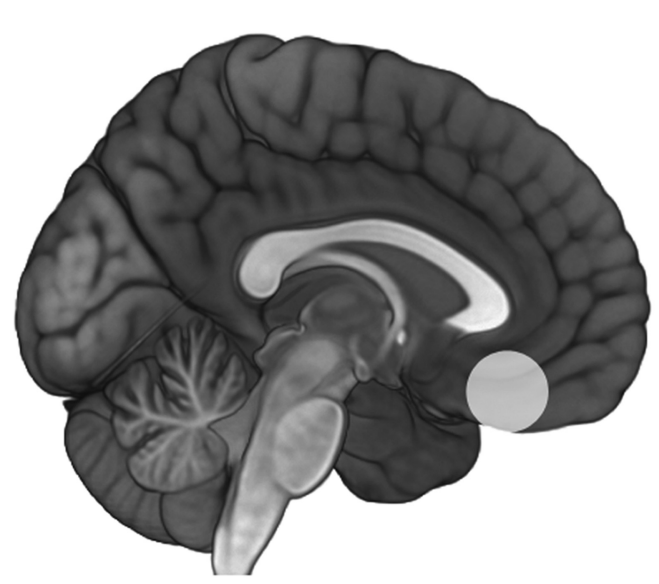

b

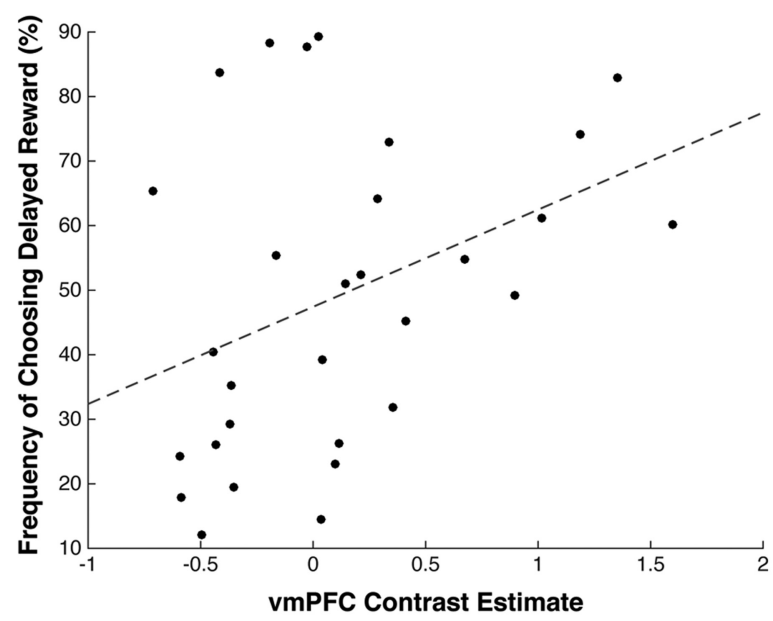

Figure 1. Increased activity for imagined juice rewards in vmPFC is associated with greater frequency of choosing to wait for larger, later monetary rewards. $\boldsymbol{a}$, The white circle outlines the 10-mm sphere in vmPFC from which BOLD data were extracted for all analyses described in Results. $\boldsymbol{b}$, The scatter plot illustrates the relationship between the contrast for imagining versus consuming juice in the vmPFC ROI and the percentage of trials where a participant chose to wait for the larger, later monetary reward. Each black dot represents one participant. The dashed gray line indicates the best linear fit to the data.

based on behavior alone and serves as a comparison for our model that incorporates the neural measure of primary reward imagination. Relative balanced accuracy rates between the two models were compared using a paired $t$ test against a null distribution derived from 10,000 permutations of the data.

In addition to our primary correlation analysis using the a priori ROI from Bray et al. (2010), we also conducted an exploratory, whole-brain analysis of the relationship between imagining juice rewards and monetary temporal discounting for completeness. Specifically, we computed between-subjects correlations for the BOLD contrast, (Imagine Juice Consume Juice), and the temporal discounting parameter, $k$, in each voxel using nonparametric permutation tests $(N=5000$ permutations $)$ using the Randomize function from the FMRIB Software Library 5.0 (FSL, FMRIB, Oxford) (Hayasaka and Nichols, 2003; Jenkinson et al., 2012). Last, for completeness, we also computed $2 \times 2$ ANOVA on action (Imagine, Consume) and liquid (Juice, Water) trial type across all participants regardless of temporal discount rate. The significance of all effects from this ANOVA was assessed after correcting for multiple comparisons at the whole-brain level using random field theory as implemented in SPM8.

\section{Results}

Self-reports of imagination are not associated with temporal discounting rates

Self-reported imagination success was not significantly correlated with monetary temporal discounting rates. Following the juice reward imagination task, participants indicated that they were able to imagine juice rewards as instructed. On average, participants reported being moderately successful in imagining the receipt of juice rewards during the Imagine trials (range $=1$, 9 ; mean $=5.97,95 \% \mathrm{CI}=5.16,6.77$; rating of $5=$ "somewhat successful") and that the experience of imagining was similar to actually consuming the juice (range $=2,9$; mean $=6.03,95 \%$ $\mathrm{CI}=5.40,6.67$; rating of $5=$ "somewhat similar)." Discount rates for future rewards were computed using a hyperbolic model based on the choices made during a separate ITC task. This parameter represents the degree to which an individual discounts or reduces the value of rewards that will be obtained at a later date; accordingly, smaller values of $k$ indicate a greater willingness to wait for delayed rewards. The discount parameters in the current sample ranged from 0.0005 to 0.0267 (mean $=0.0079$; median $=$
0.0061). Tests of the relationship between $k$ values and juice imagination success ratings $(r=0.0972 ; 95 \% \mathrm{CI}=-0.3256$, 0.4888 ; one-tailed $p=0.692)$ and similarity ratings $(r=0.0006$; $95 \% \mathrm{CI}=-0.2913,0.3529$; one-tailed $p=0.495$ ) found no evidence for an association between these behavioral measures.

\section{Neural responses to imagined reward are predictive of delay discounting rates}

To test our main hypothesis that neural activity during imagined reward receipt is associated with the degree to which future rewards are discounted, we conducted a between-subjects correlation examining the relationship between imagining juice rewards and the estimated discount parameter, $k$. This analysis was designed to test whether individuals who were more willing to wait for future monetary payments (i.e., had lower discount rates) showed increased neural activity for imagined rewards (contrast $=[$ Imagine Juice - Consume Juice $]$ ) in a region of vmPFC (Fig. 1a) previously implicated in imagining rewarding outcomes (Bray et al., 2010). There was a negative correlation between monetary temporal discount rates and the BOLD response to imagined juice rewards in this region $(r=-0.4062 ; 95 \%$ $\mathrm{CI}=-0.6072,-0.1392$; one-tailed $p=0.007)$. The correlation between imagined reward activity and temporal discounting was also significant when discounting was quantified using a modelfree measure computed simply as the percentage of time the delayed reward was chosen $(r=0.3785 ; 95 \% \mathrm{CI}=0.0861,0.6531$; one-tailed $p=0.019$ ), indicating that individuals with greater activity for imagined juice rewards were more willing to wait for delayed monetary rewards. Indeed, many participants who showed a stronger tendency to wait for the larger, later rewards had greater vmPFC activity for imagined compared with experienced juice rewards (Fig. 1b). However, we note that the effect size for the correlation between relative vmPFC activity and discounting rates is similar in the subsets of participants who have either negative $(r=-0.44)$ or positive values $(r=-0.49)$ for the contrast between imagined and consumed juice rewards.

In an additional supporting analysis, we examined whether the relationship between activity during juice imagination and temporal discounting remained when controlling for any general 

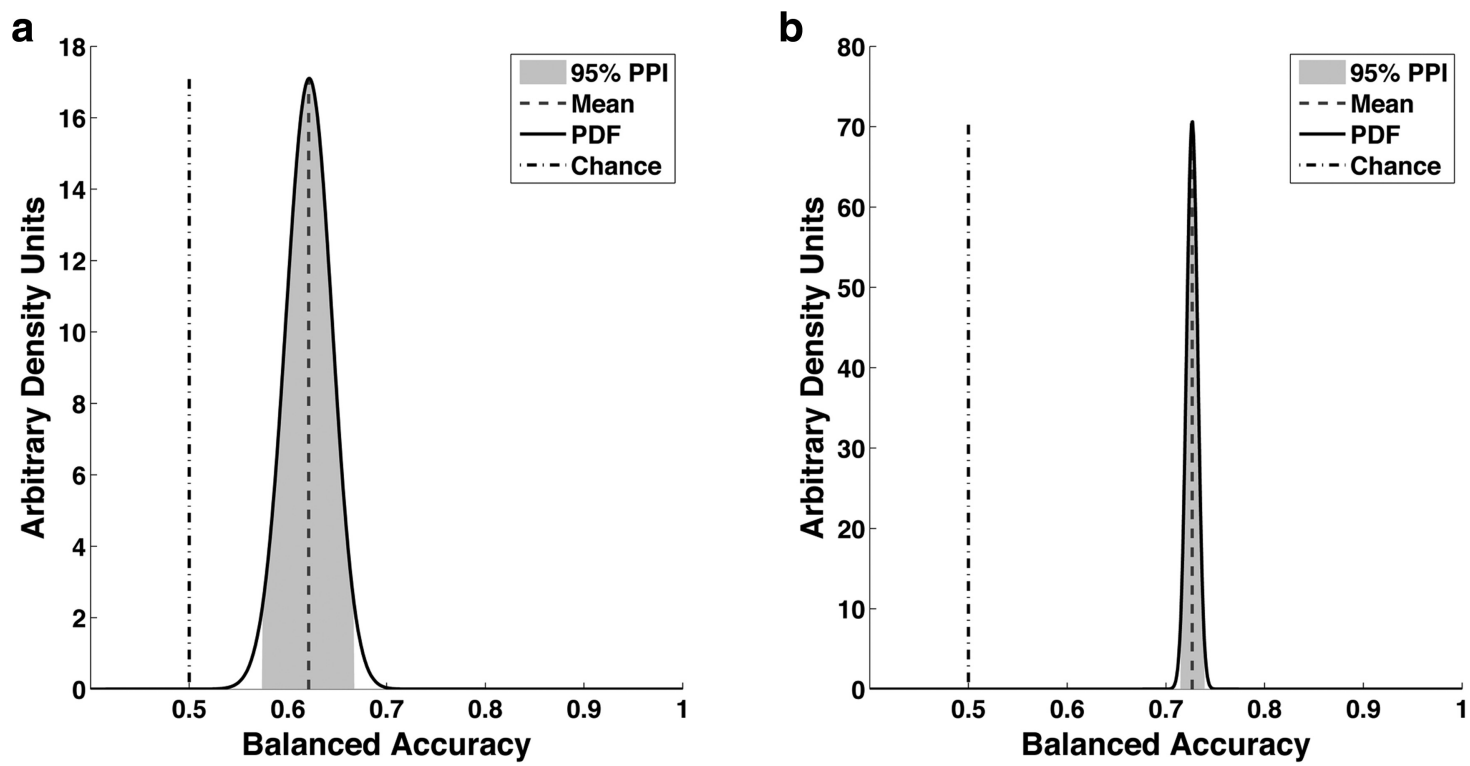

Figure 2. $\quad$ vmPFC response to imagined juice rewards predicts monetary ITC behavior both between and within individuals. $\boldsymbol{a}$, The solid black curve indicates the posterior density function (PDF) of the balanced accuracy achieved when using imagined juice responses in vmPFC to determine the relative discounting rates between participants. Light gray shading represents the $95 \%$ posterior probability interval (PPI). Dark gray dashed line indicates the mean of the posterior balanced accuracy while the dashed-dotted line depicts chance. $\boldsymbol{b}$, The solid black curve indicates the PDF of the balanced accuracy achieved when using imagined juice responses in vmPFC to predict choices on each individual trial. Light gray shading represents the $95 \%$ PPI. Dark gray dashed line indicates the mean of the posterior balanced accuracy while the dashed-dotted line depicts chance.

effects of liquid imagination and consumption. Specifically, we repeated the analysis using a difference-in-differences contrast [(Imagine Juice - Imagine Water) - (Consume Juice - Consume Water)] and found that the correlation with the discount parameter $k$ remained significant $(r=-0.3573 ; 95 \%$ $\mathrm{CI}=-0.5921,-0.0108$; one-tailed $p=0.024)$. We also tested whether the relationship between vmPFC activity and delay discounting was specific to imagined relative to experienced rewards by repeating the correlation analysis with the BOLD contrast for consuming juice versus consuming water and imagining juice versus imagining water. There was no significant correlation between discounting rates and vmPFC activity during juice versus water consumption $(r=0.2653 ; 95 \% \mathrm{CI}=-0.1201,0.5302$; two-tailed $p=0.160)$ or juice versus water imagination $(r=-0.2432$; CI $=-0.4966,0.0642$; two-tailed $p=0.179)$.

To further quantify and probe the generalizability of the relationship between vmPFC BOLD signals during imagined primary rewards and monetary temporal discounting rates, we performed an out-of-sample classification analysis to distinguish between individuals with higher and lower discount rates on the basis of their vmPFC response to imagined juice rewards. First, we computed a GLM regressing the BOLD contrast, ([Imagine Juice - Consume Juice]), against temporal discounting rates in 28 of the 30 participants. Next, we used the regression coefficients relating vmPFC BOLD signals to discounting rates in the training subset of 28 participants to predict which of the two independent test participants would be more likely to wait for delayed monetary rewards during the temporal discounting task. This process was repeated for each of the 435 unique divisions of the data into training sets of 28 and test sets of two participants. The mean balanced accuracy over all iterations was $62.10 \%$ (95\% posterior probability interval $=57.53,66.67$; Fig. $2 a)$. To examine whether there was any bias in our classification approach, we repeated the same exercise 1000 times while randomly reshuffling the discounting rate labels across participants in the training set. As expected, permuting the training labels yielded chance-level ac- curacy for this set of classifiers (mean $=50.32 \%$; $95 \% \mathrm{CI}=45.75$, 54.94). Once again, the relationship between imagined primary reward activity in vmPFC and monetary temporal discounting is similar if we replace the $k$ parameters from the hyperbolic discounting model with a model-free measure of discounting (frequency of choosing delayed rewards) in our classifier $($ mean $=64.24 \% ; 95 \%$ posterior probability interval $=59.72$, 68.73 ), indicating that this relationship is not restricted to modeling temporal discounting with a specific functional form.

One advantage of fitting temporal discounting rates with the hyperbolic model, however, is that it allows us to make predictions about whether or not an individual will chose the immediate or delayed reward when faced with a specific set of options. Thus, we tested whether the $k$ parameters estimated from the relative vmPFC response to imagined versus consumed juice rewards could be used to make out-of-sample predictions about the choice a participant made on each trial. We found that the imagination-based estimates of $k$ correctly predicted whether participants chose the immediate or delayed reward on each trial with a mean balanced accuracy of $72.66 \%$ (95\% posterior probability interval $=71.58,73.82$; Fig. $2 b$ ). At the individual level, we were able to make better than chance predictions for every participant in our sample (balanced accuracy range, 56.09\%$88.20 \%)$. This balanced accuracy rate is higher than that obtained with a benchmark algorithm that determined $k$ parameters from the choices of all other participants (mean $=69.68 \%$; $95 \%$ posterior probability interval $=68.58,70.87$; see Materials and Methods). The comparison between the two prediction schemes demonstrates that information about vmPFC activity during reward imagination relative to the direct experience of reward allows us to make better choice predictions for the test participant than the knowledge of how other participants in the sample made decisions on average $\left(t_{(29)}=2.67 ; 95 \% \mathrm{CI}=0.40,3.2 ; p=0.012\right)$. Furthermore, as would be expected, the accuracy rate of choice predictions derived from vmPFC activity during juice imagination increases relative to predictions made by the benchmark 
Table 2. Results of a whole-brain exploratory analysis testing for correlations between the BOLD contrast for imagining greater than consuming juice rewards and temporal discount rate ${ }^{a}$

\begin{tabular}{lcrrrr}
\hline Region & Cluster size $^{b}$ & $x$ & $y$ & \multicolumn{1}{c}{$z$} & ${\text { Peak } t^{c}}^{\text {Cerebellum/brainstem }}$ \\
\hline Rectal/medial orbitofrontal gyrus & 148 & -12 & -33 & -27 & 4.85 \\
Lateral orbitofrontal cortex & 77 & 0 & 27 & -30 & 4.29 \\
Medial frontal gyrus & 15 & 45 & 42 & -18 & 4.02 \\
& 10 & 9 & 54 & 21 & 3.59
\end{tabular}

${ }^{a}$ This table reports all clusters containing $\geq 10$ voxels at a voxel-wise significance threshold of $p<0.001$. None of the regions listed here were significant after correcting for multiple comparisons at the whole-brain level.

${ }^{b}$ Cluster size indicates the number of voxels at an isotropic resolution of $3 \mathrm{~mm}$.

' $t$ statistics were computed using 5000 permutations of the data.

Table 3. Regions showing significantly different BOLD response between imagination and consumption trials ${ }^{a}$

\begin{tabular}{lcrrrr}
\hline Region & Cluster size $^{b}$ & $x$ & $y$ & $z$ & Peakt \\
\hline $\begin{array}{l}\text { Imagine - Consume } \\
\quad \text { Parietal lobe/occipital lobe }\end{array}$ & 4926 & 51 & -75 & 42 & 6.15 \\
$\quad$ Dorsolateral prefrontal cortex/anterior & 3790 & -39 & 48 & 9 & 6.26 \\
$\quad$ cingulate cortex & & & & & \\
$\quad$ Occipital pole & 31 & -3 & -105 & -6 & 3.31 \\
$\quad$ Lateral parietal lobe & 3 & -51 & -72 & 36 & 4.23 \\
Consume - Imagine & & & & & \\
$\quad$ Insula & 276 & -39 & -6 & 3 & 7.2 \\
Insula & 189 & 39 & -3 & 3 & 7.69 \\
\hline
\end{tabular}

${ }^{a}$ All regions are statistically significant at $p<0.05$ after whole-brain correction for multiple comparisons.

${ }^{b}$ Cluster size indicates the number of voxels at an isotropic resolution of $3 \mathrm{~mm}$.

model as the independent test participant's discount rate deviates from the median discount rate of the entire sample $(r=0.4387$; $95 \% \mathrm{CI}=0.2177,0.6277 ; p=0.010)$. Each participant's deviation from the median discount rate was computed as |participant $k-$ group median $k \mid$ with all $k$ parameters estimated directly from participant choice data. Thus, the degree to which the relative level of vmPFC activity during juice reward imagination improves our ability to predict monetary temporal discounting choices increases as the test participant's choice behavior diverges from the decisions made by the other participants in the sample.

In addition to our primary analysis using the a priori vmPFC ROI from Bray et al. (2010), for completeness, we also examined the relationship between imagining juice rewards and monetary temporal discounting using an exploratory, whole-brain approach. However, although regions including medial and lateral orbitofrontal cortex were correlated with discount parameters at individual voxel level thresholds of $p<0.001$, none of these results remained significant after correction for multiple comparisons at the whole-brain level. We have included the full list of regions in Table 2 to facilitate future meta-analyses but refrain from any interpretation of these results. Finally, we also report the results of the main effect of action type (Imagine vs Consume) in Table 3. Neither the main effect of liquid type (Juice vs Water) nor the interaction between action (Imagine, Consume) and liquid (Juice, Water) was significant in any voxels after correcting for multiple comparisons. We report the main effects of action as paired $t$ tests in Table 3 to convey the direction of the effect for each region.

\section{Discussion}

We investigated the brain-behavior associations between the neural representations of imagined primary reward and monetary temporal discounting behavior. Consistent with the hypothesis that individuals who generate greater neural responses for imagined rewards would show reduced discounting of future re- wards, we found that temporal discounting rates were negatively correlated with brain activity in a region of vmPFC previously associated with the processing of both real and imagined rewards (Bray et al., 2010; Bartra et al., 2013; Clithero and Rangel, 2014). These data suggest that the relative strength of the neural representation of imagined rewards may drive individual differences in real-world economic decision making. Moreover, this neural measure of reward imagination was able to significantly predict future monetary discounting behavior both between and within individuals, whereas self-reported measures of imagination success showed no significant association with discount rate.

Neural activity in vmPFC has been repeatedly linked to the processing of primary and secondary reward values at both the time of choice and consumption (see meta-analyses by Bartra et al., 2013; Clithero and Rangel, 2014). Bray et al. (2010) showed that vmPFC is activated when imagining rewards, but, importantly, not when imagining visual motion or motor actions. Moreover, prior expectations have been found to influence vmPFC responses to the receipt of rewards (Plassmann et al., 2008). Plassmann et al. (2008) showed that cognitive expectations could modulate responses in vmPFC to reward consumption and that these vmPFC responses correlated with subjective pleasantness ratings. These previous results are consistent with the idea that vmPFC is involved in computing the pleasantness or utility of outcomes and can be influenced by both the presence and expectation of rewards. In light of our current data, this suggests that enhanced vmPFC activation during the imagination and simulation of rewarding experiences, regardless of when such rewards may occur, is associated with placing greater value on future reward outcomes.

How might greater vmPFC activity during reward imagination relative to consumption lead to reduced discounting of future rewards? Although we cannot definitively establish that vmPFC activity during juice imagination in our task is a representation of the imagined pleasantness of the juice, such a role for vmPFC would be consistent with the extensive literature on experienced and expected value representations in this region (discussed above) as well as the specific actions performed (liquid consumption or imagination) while vmPFC activity was measured. Moreover, the fact that the relationship between vmPFC activity and temporal discounting holds when the vmPFC signal is instead computed as the relative difference between juice reward and water during imagination and consumption (i.e., the contrast [(Imagine Juice - Imagine Water) - (Consume Juice Consume Water)]) further indicates that the link between vmPFC activity and temporal discounting might be a representation of the imagined pleasantness of the liquids rather than a more general aspect of imagination. Our data do directly show that many of the shallowest discounters (i.e., those who place the highest value on larger delayed rewards) actually have greater vmPFC activity during reward imagination than consumption. If we assume that vmPFC activity is an index of imagined reward qualities, then a natural interpretation of our findings is that these individuals overestimate expected value when imagining delayed rewards, which results in a relatively higher value for delayed compared with immediately experienced rewards. The same logic would also apply in the opposite direction for those with relatively less vmPFC activity during reward imagination compared with consumption. Again, assuming that vmPFC activity is an index of imagined reward qualities, such individuals may underestimate the value of imagined rewards and therefore more steeply discount rewards they imagine experiencing in the future. We therefore suggest that the level of vmPFC activity 
during reward imagination compared with consumption serves as an index for the degree to which an individual overestimates or underestimates the pleasantness of reward consumption when imagining an experience. We hasten to note, however, that our assumption that vmPFC activity is a representation of imagined reward value remains to be tested directly in future studies that include several imagined rewards of differing value levels, a feature not present in the current design. Although we cannot directly show that vmPFC activity reflects the value of imagined rewards, we clearly demonstrate that activity in this ROI for imagined versus presently experienced rewards is closely associated with the degree to which future rewards are discounted.

Our data complement and extend previous findings on the role of imagery and time perception in ITC. Two studies (Peters and Büchel, 2010; Benoit et al., 2011) have shown that enhancing the concreteness and vividness (i.e., aiding imagination) of a future outcome at the time of choice reduced discounting rates. We demonstrate an association between an individual's imagined experience of a reward (i.e., consuming juice right now) in the absence of choice and monetary discounting behavior in a separate ITC task. Unlike previous studies that enhanced the vividness of future monetary outcomes during choice to reduce discounting, our data link individual differences in reward imagination across modality (juice vs money) and time (now vs future) to discount rates in a separate choice task. These findings suggest that a fundamental ability or propensity to imagine the utility of both present and future outcomes may reduce the rate at which future rewards are discounted. However, we note that no study has yet established that individual differences in measures of imagination at the behavioral or neural level are an index of imagination ability rather than differences in motivation, attention, fatigue, or other factors, and distinguishing between these factors is an important goal for future research. Another recent study (Cooper et al., 2013) has shown that BOLD responses in the ventral striatum and vmPFC during the evaluation of temporal durations (e.g., how long is $28 \mathrm{~d}$ ?), also in the absence of choice, are correlated with temporal discounting rates. Thus, individual differences in the neural representation of imagined reward consumption and the perception of delays are predictive of individual differences in temporal discounting behavior in the absence of choice. Together, these results indicate that individual differences in the neural representation of imagined rewards and time are an important factor in the observed heterogeneity in reward discounting behavior across individuals.

We believe it is worth emphasizing that both reward imagination and delay perception predict ITC behavior when measured in the absence of actual choices, making them potentially useful diagnostic measures when choice behavior cannot be collected for ethical or practical reasons. The psychological and neurobiological processes of both reward imagination and time perception present intriguing targets for future research on temporal discounting behaviors and potential avenues for therapeutic interventions aimed at boosting the value placed on future outcomes.

\section{References}

Ainslie G (2001) Breakdown of will. Cambridge, MA: Cambridge UP. Bartra O, McGuire JT, Kable JW (2013) The valuation system: a coordinatebased meta-analysis of BOLD fMRI experiments examining neural correlates of subjective value. Neuroimage 76:412-427. CrossRef Medline Benoit RG, Gilbert SJ, Burgess PW (2011) A neural mechanism mediating the impact of episodic prospection on farsighted decisions. J Neurosci 31:6771-6779. CrossRef Medline

Berns GS, Laibson D, Loewenstein G (2007) Intertemporal choice: toward an integrative framework. Trends Cogn Sci 11:482-488. CrossRef Medline

Bray S, Shimojo S, O’Doherty JP (2010) Human medial orbitofrontal cortex is recruited during experience of imagined and real rewards. J Neurophysiol 103:2506-2512. CrossRef Medline

Brodersen KH, Ong CS, Stephan KE, Buhmann JM (2010) The balanced accuracy and its posterior distribution. 2010 20th International Conference on Pattern Recognition, 3121-3124.

Clithero JA, Rangel A (2014) Informatic parcellation of the network involved in the computation of subjective value. Soc Cogn Affect Neurosci 9:1289-1302. CrossRef Medline

Cooper N, Kable JW, Kim BK, Zauberman G (2013) Brain activity in valuation regions while thinking about the future predicts individual discount rates. J Neurosci 33:13150-13156. CrossRef Medline

Daniel TO, Stanton CM, Epstein LH (2013) The future is now: reducing impulsivity and energy intake using episodic future thinking. Psychol Sci 24:2339-2342. CrossRef Medline

Deichmann R, Gottfried JA, Hutton C, Turner R (2003) Optimized EPI for fMRI studies of the orbitofrontal cortex. Neuroimage 19:430-441. CrossRef Medline

Green L, Myerson J (2004) A discounting framework for choice with delayed and probabilistic rewards. Psychol Bull 130:769-792. CrossRef Medline

Hare TA, Hakimi S, Rangel A (2014) Activity in dlPFC and its effective connectivity to vmPFC are associated with temporal discounting. Front Neurosci 8:50. CrossRef Medline

Hayasaka S, Nichols TE (2003) Validating cluster size inference: random field and permutation methods. Neuroimage 20:2343-2356. CrossRef Medline

Jenkinson M, Beckmann CF, Behrens TE, Woolrich MW, Smith SM (2012) FSL. Neuroimage 62:782-790. CrossRef Medline

Kirby KN, Maraković NN (1995) Modeling myopic decisions: evidence for hyperbolic delay-discounting within subjects and amounts. Organ Behav Hum Decis Process 64:22-30. CrossRef

Lebreton M, Bertoux M, Boutet C, Lehericy S, Dubois B, Fossati P, Pessiglione M (2013) A critical role for the hippocampus in the valuation of imagined outcomes. PLoS Biol 11:e1001684. CrossRef Medline

MacKillop J, Amlung MT, Few LR, Ray LA, Sweet LH, Munafo MR (2011) Delayed reward discounting and addictive behavior: a meta-analysis. Psychopharmacology (Berl) 216:305-321. CrossRef Medline

Moffitt TE, Arseneault L, Belsky D, Dickson N, Hancox RJ, Harrington H, Houts R, Poulton R, Roberts BW, Ross S, Sears MR, Thomson WM, Caspi A (2011) A gradient of childhood self-control predicts health, wealth, and public safety. Proc Natl Acad Sci U S A 108:2693-2698. CrossRef Medline

Peters J, Büchel C (2010) Episodic future thinking reduces reward delay discounting through an enhancement of prefrontal-mediotemporal interactions. Neuron 66:138-148. CrossRef Medline

Peters J, Büchel C (2011) The neural mechanisms of inter-temporal decision-making: understanding variability. Trends Cogn Sci 15:227-239. CrossRef Medline

Plassmann H, O’Doherty J, Shiv B, Rangel A (2008) Marketing actions can modulate neural representations of experienced pleasantness. Proc Natl Acad Sci U S A 105:1050-1054. CrossRef Medline

Radu PT, Yi R, Bickel WK, Gross JJ, McClure SM (2011) A mechanism for reducing delay discounting by altering temporal attention. J Exp Anal Behav 96:363-385. CrossRef Medline

Shamosh NA, Deyoung CG, Green AE, Reis DL, Johnson MR, Conway AR, Engle RW, Braver TS, Gray JR (2008) Individual differences in delay discounting: relation to intelligence, working memory, and anterior prefrontal cortex. Psychol Sci 19:904-911. CrossRef Medline

Suddendorf T, Corballis MC (2007) The evolution of foresight: what is mental time travel, and is it unique to humans? Behav Brain Sci 30:299313; discussion 313-351. CrossRef Medline

van den Bos W, McClure SM (2013) Towards a general model of temporal discounting. J Exp Anal Behav 99:58-73. CrossRef Medline 